\title{
Energy Efficiency in the Wideband Regime *
}

\author{
Guowang Miao ${ }^{\dagger, b}$ and Anders Vastberg ${ }^{\dagger}$ \\ $\dagger$ KTH Royal Institute of Technology
}

\begin{abstract}
In this paper we discuss optimal energy-efficient communications in the wideband regime, where the signal bandwidth is infinite and the channel is flat. A multiple-hop system with one sender, one receiver, and multiple relays is considered. We discover the globally optimal link adaptation as well as relay deployment strategies such that the whole system can run with the highest energy efficiency. The technologies proposed can be used in various communication systems, such as the deployment and communication of wired core networks and wireless broadband relay backhauls, to improve their energy efficiencies.

Index Terms-interference, energy efficiency, power optimization, OFDM, non-cooperative power control
\end{abstract}

\section{INTRODUCTION}

Energy efficiency is increasingly important for communications and networks because of both climate and cost issues. Information and communication technology (ICT) plays an important role in global greenhouse gas emissions since the amount of energy consumed by ICT increases dramatically to meet with the explosive growing service requirements [1]. It is shown that nowadays the total energy used by the infrastructure of cellular networks, wired networks, and Internet takes up more than $3 \%$ of the worldwide electric energy consumption [2]. In addition, this amount of energy is expected to increase rapidly in the future.

The exponential relationship between rate and power in Shannon capacity indicates that energy efficiency can only be achieved with infinite bandwidth or infinitely small data rate. But this qualitative analysis considers only transmission energy and ignores practical issues like other types of power consumption, such as those used for operating circuit and signal processing [3]. Therefore energy-efficient network design should find tradeoff between transmission energy and energy overhead like circuit energy. This tradeoff exists in almost all aspects of wireless networks, from individual transceivers [4]-[6] to cellular network deployments [7], [8]. For example in [4], a unique globally optimal power allocation and link adaptation is discovered for multiple input and multiple output (MIMO) and orthogonal frequency division multiplexing (OFDM) transmission and this scheme automatically balances the energy consumption between the the circuit power and the transmission powers on all subchannels. When there are multiple users in the network, the network resources should also be managed such that

b Corresponding author. Email: guowangmiao@gmail.com. Address: Electrum 229, Kista, Stockholm, 16440, Sweden the overall network energy efficiency is maximized rather than single users [3], [6], [9], [10]. A thorough tutorial can be found in [3], which discusses energy-efficient resource management in time, frequency, and space domains. In addition to single cell resource management, energy efficiency should also be considered from the multi-cell aspect [11][13]. In [11], [12], it is observed that power optimization is important in both energy efficiency improvement and intercell interference control. Besides, energy-efficient power control not only improves network energy efficiency by also spectral efficiency because its conservative nature of power allocation automatically alleviates inter-cell interference to improve overall network throughput.

In this paper, we study energy-efficient communications in the wideband regime, where the signal bandwidth is large and the channel is flat. Examples include wired communications using copper or fiber optics where the bandwidth is almost infinite and the channel is flat and line-of-sight (LOS) wireless backhaul communications with multi-gigahertz bandwidth [14]. For instance, the wireless channels around $60 \mathrm{GHz}$ do not exhibit rich multipath. The non-line-of-sight (NLOS) components suffer from tremendous attenuation and the channel is almost flat [14]. In addition a broad spectrum, e.g. several gigahertz, can be used at $60 \mathrm{GHz}$ to achieve a high data rate for backhaul communications. Therefore a backhaul system using $60 \mathrm{GHz}$ carrier frequency can have large signal bandwidth and flat channel.

The scenario we will focus on would be a point-to-point link with multiple relays in between. The question we will answer is what would be the global optimal link adaptation and relay deployment strategy such that the whole system is the most energy efficient. This strategy can be used in many applications, e.g. core network deployment or wireless relay backhaul, to improve their energy efficiencies. The paper is structured as follows. In Section II we present the system model. In Section III, we discuss energy-efficient communication without any relay. In Sections IV, V, and VI, we discuss the optimal link adaptation as well relay deployment strategies assuming an arbitrary number of relays. Finally we conclude the paper in Section VIII.

\section{System Model}

Consider a multi-hop system with one sender, one receiver, and $K$ relays, as illustrated in Fig. 1. The data is sent from the sender to the first relay, which decodes the data 
Fig. 1: System Model.

and forward it to the second relay. This process repeats until the data reaches the receiver. All transmissions experience additive white Gaussian noise (AWGN) channels and the data rate is given by

$$
\begin{aligned}
& \quad R_{i}=B \log _{2}\left(1+\frac{P_{i} \xi_{i} h_{i}}{B N_{o}}\right), i=0,1, \ldots, K, \\
& \text { s.t. } \quad P_{i} \leq P_{m i},
\end{aligned}
$$

where $B$ denotes the signal bandwidth, $P_{i}$ the transmission power, $\xi_{i}$ the power amplifier efficiency, $h_{i}$ the channel power gain, $N_{o}$ the noise spectral density, and $P_{m i}$ the power limit. The transmission of the sender is indexed with 0 while the transmission of the $i$ th relay indexed with $i$. In the following we may refer to the transmitter and relays as user $i$ according to their indices. In the wideband regime, assume $B \rightarrow \infty$, then

$$
R_{i}=\frac{P_{i} \xi_{i} h_{i}}{N_{o} \ln 2} .
$$

Note that all relays work in the full-duplex mode because their data reception and transmission can be easily separated by e.g. frequency-division duplexing (FDD). The above approximation (2) still holds when $B$ is divided into half. In addition to transmission power, each device also consumes circuit power for computation purposes, e.g. signal processing, filtering, and encoding and decoding [4], and the total power consumption is

$$
\hat{P}_{i}=P_{i}+P_{c i},
$$

where $P_{c i}$ denotes the circuit power. The circuit power consumption of the receiver is defined as $P_{r}$. The energy efficiency of the system, $u$, is defined as the total number of bits that can be transmitted consuming a certain amount of energy, or equivalently, the network throughput divided by the total network power consumption [4], i.e.

$$
u=\frac{R}{\sum_{i}\left(P_{i}+P_{c i}\right)+P_{r}},
$$

where $R$ denotes the achieved data rate. In the following, we study the optimal link adaptation as well as relay deployment strategy such that the whole system is the most energy efficient.

\section{Direct EnERgy-EFficient CommunicAtions}

When there is no relay and the sender transmits data directly to the receiver, the transmitter wants to do link adaptation such that its energy efficiency is maximized, i.e. the power is given by

$$
\begin{aligned}
& P_{0}^{*}=\arg \max _{P_{0}} \frac{R_{0}}{P_{0}+P_{c 0}+P_{r}} \\
& =\arg \max _{P_{0}} \frac{\frac{P_{0} \xi h_{i}}{N_{o} \ln 2}}{P_{0}+P_{c 0}+P_{r}} \\
\text { s.t. } \quad & P_{0} \leq P_{m 0} .
\end{aligned}
$$

The energy efficiency increases with the transmission power and therefore the highest power should always be used, i.e.

$$
P_{0}^{*}=P_{m 0} .
$$

The highest energy efficiency is

$$
u^{*}=\frac{P_{m 0} \xi h_{i}}{\left(P_{m 0}+P_{c 0}+P_{r}\right) N_{o} \ln 2} .
$$

Theoretically, if there is no power limit, i.e. $P_{m 0} \rightarrow \infty$, the energy efficiency upper bound is

$$
u^{*}=\frac{\xi h_{i}}{N_{o} \ln 2} .
$$

The energy efficiency in (7) indicates five ways of improving energy efficiency from an implementation perspective, that is,

- increasing the power limit (mainly the linear range of the power amplifier),

- increasing the power amplifier efficiency,

- increasing the channel gain,

- reducing the circuit power of both the transmitter and receiver,

- and reducing the thermal noise.

\section{ENERGy-EFFICIENT RELAy LINK ADAPTATION}

In this section, we assume an arbitrary number of relays with given channels and discuss the optimal energy-efficient transmission strategy.

The overall achieved data rate of the system must be

$$
R=\min _{i} R_{i} .
$$

This also indicates that all transmissions by the sender and relays should be at the same rate $R$, as otherwise, any link with a higher rate will waste its energy consumption. Therefore, the power of user $i$ is

$$
P_{i}=\frac{R N_{o} \ln 2}{\xi_{i} h_{i}} .
$$

The overall system energy efficiency is

$$
\begin{aligned}
u & =\frac{R}{\sum_{i}\left(P_{i}+P_{c i}\right)+P_{r}} \\
& =\frac{R}{\sum_{i}\left(\frac{R N_{o} \ln 2}{\xi_{i} h_{i}}+P_{c i}\right)+P_{r}} .
\end{aligned}
$$

The energy efficiency $u$ increases with $R$. Therefore the 
highest data rate should be used and

$$
R^{*}=\min _{i} \frac{P_{m i} \xi_{i} h_{i}}{N_{o} \ln 2} .
$$

User $i$ will use just enough power to achieve $R^{*}$ and the transmission power should be

$$
P_{i}=\frac{R^{*} N_{o} \ln 2}{\xi_{i} h_{i}} .
$$

If there is no power limit, the energy efficiency upper bound is given by

$$
u^{*}=\frac{1}{\sum_{i}\left(\frac{1}{\xi_{i} h_{i}}\right)} \frac{1}{N_{o} \ln 2} .
$$

\section{ENERGy-EFFicient RELAy DEPLOYMENT}

In this section, we study how to deploy relays such that the whole system is the most energy efficient assuming all users transmit with a fixed power. Define the distance between the sender and receiver as $d$. We need to find the optimal deployment positions of all relays.

Consider only path loss and in wideband wireless communications, the channel power gain is given by

$$
h_{i}=\frac{h_{o}}{d_{i}^{\alpha}},
$$

where $d_{i}$ is the link distance of user $i, \alpha$ the path loss exponent, and $h_{o}$ the gain at the unit distance. For wired communications, the signal attenuation usually grows exponentially with the distance, e.g. $h_{i}=\frac{h_{o}}{e^{\alpha d_{i}}}[15]$, [16]. In the following, we focus on wireless communications because of page limit. However, all theories developed in the following sections can be easily extended for wired cases since both $d_{i}^{\alpha}$ and $e^{\alpha d_{i}}$ are increasing and strictly-convex functions.

The overall achieved data rate of the system is

$$
R=\min _{i} R_{i}=\min _{i} \frac{P_{i} \xi_{i} h_{o}}{d_{i}^{\alpha} N_{o} \ln 2} .
$$

Since the transmission power is fixed for all users, maximizing the energy efficiency $u$ is the same as maximizing the rate $R$ and the objective would be

$$
\begin{aligned}
& \max R=\max \min _{i} \frac{P_{i} \xi_{i} h_{o}}{d_{i}^{\alpha} N_{o} \ln 2}, \\
& \text { s.t. } \quad \sum_{i} d_{i}=d .
\end{aligned}
$$

There is a unique optimal solution to problem (17) and for the optimal solution, different users should achieve the same data rate, i.e.

$$
\frac{P_{i} \xi_{i} h_{o}}{d_{i}^{* \alpha} N_{o} \ln 2}=\frac{P_{j} \xi_{j} h_{o}}{d_{j}^{* \alpha} N_{o} \ln 2}, \forall i \neq j,
$$

as otherwise, the user with a higher data rate than the minimum rate should extend its link distance such that the distance for the user with the lowest rate can reduce its link distance to achieve a higher data rate. Therefore we have

$$
\frac{d_{i}^{*}}{\sqrt[\alpha]{P_{i} \xi_{i}}}=\frac{d_{j}^{*}}{\sqrt[\alpha]{P_{j} \xi_{j}}}
$$

and the optimal deployment strategy is

$$
d_{i}^{*}=\frac{\sqrt[\alpha]{P_{i} \xi_{i}}}{\sum_{j} \sqrt[\alpha]{P_{j} \xi_{j}}} d .
$$

Correspondingly, the highest energy efficiency of the system is

$$
u^{*}=\frac{h_{o}\left[\sum_{i}\left(P_{i} \xi_{i}\right)^{\frac{1}{\alpha}}\right]^{\alpha}}{d^{\alpha}\left(\sum_{i}\left(P_{i}+P_{c i}\right)+P_{r}\right) N_{o} \ln 2} .
$$

With the optimal deployment strategy, the link distance of each user will be adjusted such that its data rate is the same as all other users.

\section{ENERGy-EFFicIENT RELAy DEPLOYMENT AND LINK ADAPTATION}

In this section we discuss the optimal energy-efficient relay deployment with link adaptation considering the power limits.

The optimal deployment in this case should still satisfy the condition in Section V because the deployment strategy in (20) is optimal for all transmission power settings. The remaining question is what would be the optimal transmission powers of all users given the power limits.

The problem is to find the optimal transmission powers to maximize (21). From now on, let $u$ denote the expression in (21). We have the following objective

$$
\begin{aligned}
& \max _{\mathbf{P}} u=\frac{h_{o}\left[\sum_{i}\left(P_{i} \xi_{i}\right)^{\frac{1}{\alpha}}\right]^{\alpha}}{d^{\alpha}\left(\sum_{i}\left(P_{i}+P_{c i}\right)+P_{r}\right) N_{o} \ln 2} \\
& \text { s.t. } \quad P_{i} \leq P_{m i} .
\end{aligned}
$$

where the vector $\mathbf{P}=\left[P_{0}, P_{1}, \ldots, P_{K}\right]$. Similar to the proof in [4], it can be easily proven that the objective function is strictly quasi-concave in $\mathbf{P}$ and there is a unique globally optimal power setting. Furthermore, $u$ first strictly increases in $P_{i}$ and then decreases in it. Therefore user $i$ will use either the peak power $P_{m i}$ or the power value at which the first-order derivative of $u$ is zero. Summarizing the above, we have the following necessary and sufficient condition for the globally optimal relay deployment and link adaptation and the maximum energy efficiency.

Theorem 1. There exists a unique globally optimal relay deployment $\mathbf{d}^{*}=\left[d_{0}^{*}, \ldots, d_{K}^{*}\right]$ and link adaptation, $\mathbf{P}^{*}$, where

$$
d_{i}^{*}=\frac{\sqrt[\alpha]{P_{i}^{*} \xi_{i}}}{\sum_{j} \sqrt[\alpha]{P_{j}^{*} \xi_{j}}} d .
$$

and

$$
\text { 1) if } P_{m i}<\left[\frac{\xi_{i}\left(P_{\text {idle }}+\sum_{j \neq i} P_{j}^{*}\right)^{\alpha}}{\left(\sum_{j \neq i}\left(P_{j}^{*} \xi_{j}^{*}\right)^{\frac{1}{\alpha}}\right)^{\alpha}}\right]^{\frac{1}{\alpha-1}} \text {, then } P_{i}^{*}=P_{m i} \text {; }
$$


2) otherwise

$$
P_{i}^{*}=\left[\frac{\xi_{i}\left(P_{\text {idle }}+\sum_{j \neq i} P_{j}^{*}\right)^{\alpha}}{\left(\sum_{j \neq i}\left(P_{j}^{*} \xi_{j}^{*}\right)^{\frac{1}{\alpha}}\right)^{\alpha}}\right]^{\frac{1}{\alpha-1}} .
$$

Here $P_{\text {idle }}=\sum_{i} P_{c i}+P_{r}$, the total power except transmission power. The maximum energy efficiency of the system is

$$
u^{*}=\frac{\left[h_{o} \sum_{i}\left(P_{i}^{*} \xi_{i}\right)^{\frac{1}{\alpha}}\right]^{\alpha}}{d^{\alpha}\left(\sum_{i}\left(P_{i}^{*}+P_{c i}\right)+P_{r}\right) N_{o} \ln 2} .
$$

Assume no power limit and $\xi_{i}=\xi_{j}=1, \forall i \neq j$. Then $P_{i}^{*}=P_{j}^{*}$ according to (24). The maximum energy efficiency is

$$
u^{*}=\frac{h_{o} P_{0}^{*}(K+1)^{\alpha}}{d^{\alpha}\left((K+1) P_{0}^{*}+P_{i d l e}\right) N_{o} \ln 2} .
$$

$u^{*}$ increases in $P_{0}^{*}$. Therefore we have the upper bound of $u^{*}$ when $P_{0}^{*} \rightarrow \infty$, i.e.

$$
u^{* *}=\frac{h_{o}(K+1)^{\alpha-1}}{d^{\alpha} N_{o} \ln 2} .
$$

This indicates that without power limit, the energy efficiency of the system can always be improved with more relays. An intuitive explanation is that with infinitely high data rate, the time sending each bit can be infinitely small and the circuit energy consumption is negligible. The system energy efficiency is therefore almost solely determined by the transmission energy. As the energy consumption increases exponentially with the distance, more relays will always help reducing the communication distances and therefore improving the energy efficiency exponentially. However this conclusion may no longer be valid when there are power limits. With power limits, the data rate will be limited and circuit energy consumption is not negligible. Whether an additional relay can improve system energy efficiency will depend on how much additional circuit power the relay consumes. However we can always use Theorem 1 to compare the energy efficiency when the system has different sets of relays and decide how many relays would be the most energy efficient.

Theorem 1 does not give the explicit $\mathbf{P}^{*}$ and numerical algorithms are needed to search for it. A similar issue has been thoroughly studied in [4] to find the optimal energyefficient link adaptation for MIMO and OFDM communications. The difference in this paper is the per-element power limits, $\left\{P_{m i}\right\}$. If the power limits are sufficiently large, the binary search assisted ascent (BSAA) algorithm in [4] can be used here too. Otherwise, we can modify BSAA slightly to reflect the consideration of per-element power limits. The improved BSAA for finding the optimal power setting is summarized in Table I.

\section{Simulations}

$60 \mathrm{GHz}$ wireless channels have very high bandwidth and limited transmission power, which means that the wideband

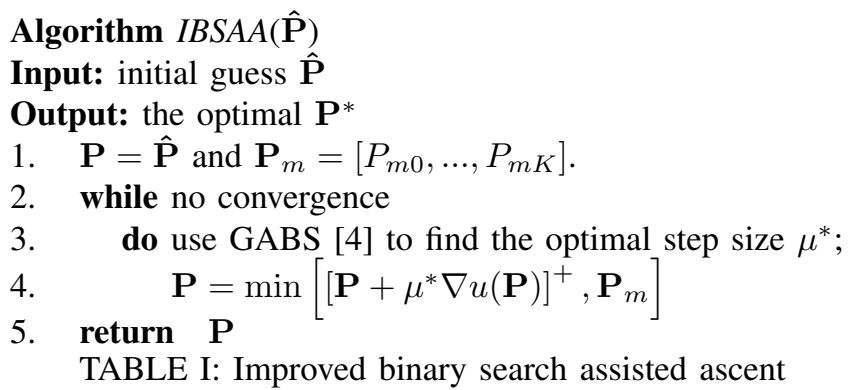

TABLE II: Simulation Parameters

\begin{tabular}{|c|c|}
\hline Maximum Tx power, $P_{m}$ & $20 \mathrm{~mW}$ \\
\hline Path loss at $1 \mathrm{~m}, P L_{0}$ & $57.5 \mathrm{~dB}$ \\
\hline Path loss exponent, $\alpha$ & 2.5 \\
\hline Noise figure, $N F$ & $6 \mathrm{~dB}$ \\
\hline Noise spectral density, $N_{o}$ & $-204 \mathrm{~dB} / \mathrm{Hz}$ \\
\hline Implementation Loss, $I L$ & $6 \mathrm{~dB}$ \\
\hline Antenna Gain, $G_{t}, G_{r}$ & $30 \mathrm{~dB}$ \\
\hline Bandwidth, B & $1.5 \mathrm{GHz}$ \\
\hline Circuit power for the transmitter, $P_{c}$ & $0.2 \mathrm{~W}$ \\
\hline Circuit power for the receiver, $P_{r}$ & $0.2 \mathrm{~W}$ \\
\hline Power Amplifier efficiency, $\xi$ & 0.1 \\
\hline
\end{tabular}

approximation is valid. We have looked at a short distance $60 \mathrm{GHz}$ radio links and used the system model in Figure 1. The simulation parameters are summarized in Table II, most of which are based on the parameters in [17]. The circuit power and power amplifier efficiency are estimated from comparisons to similar low power transmitters. The antenna gain, noise figure, and implementation loss are included in the constant $h_{o}$ in equation (15) and the channel power gain is expressed as follows,

$$
h(d)=\frac{G_{t} G_{r}}{P L_{0} \cdot I L \cdot N F \cdot d^{\alpha}} .
$$

Fig. 2: Energy-efficiency and distance with one relay.

In Figure 2, we show the energy efficiency as a function of distance for a direct link and for a link with one relay. The upper bound is for the link with one relay and no power limit. Using relays improves the energy efficiency as the decreased path loss leads to increased maximum data rate. This effect is much larger compared to the increased power 
Fig. 3: Energy-efficiency and distance.

consumption due the relay transmission and circuit power. Figure 3 shows that the energy efficiency increases with the number of relays, but the gain diminishes with higher numbers of relays, in which case more relays is needed for the same effect. This diminishing return is shown more clearly Figure 4. In Figure 5 it is shown that increased circuit power of relays reduces the benefit on energy efficiency of using multiple relays.

Fig. 4: Energy-efficiency and number of relays.

Fig. 5: Energy-efficiency and circuit power.

\section{CONCLUSION}

In this paper we have discussed optimal energy-efficient communications in the wideband regime. Specifically we have discovered the globally optimal link adaptation as well as relay deployment strategies such that a multi-hop system can run with the highest energy efficiency. We have shown that if only link adaptation is used, all users should transmit with the highest data rate necessary so that the circuit energy consumption can be minimized. When all users transmit with fixed power, the relays should be deployed such that each link achieves the same data rate. If both the deployment and power can be adapted, we have discovered the unique and globally optimal strategy that automatically balances the contribution to power consumption from transmission, circuit operation, distance, and power amplifiers. The technologies proposed can be used in various communication systems, such as the deployment and communication of wired core networks and wireless broadband relay backhauls, to improve their energy efficiencies.

\section{REFERENCES}

[1] O. Blume, D. Zeller, and U. Barth, "Approaches to energy efficient wireless access networks," in ISCCSP 2010, Limassol, Cyprus, Mar. 2010, pp. $1-5$.

[2] G. P. Fettweis and E. Zimmermann, "ICT energy consumptiontrends and challenges," in Proc. 11th Int. Symp. Wireless Personal Multimedia Commun., Sep. 2008.

[3] G. W. Miao, N. Himayat, Y. Li, and A. Swami, "Cross-layer optimization for energy-efficient wireless communications: A survey," to appear in Wireless Commun. and Mobile Computing $J$.

[4] G. W. Miao, N. Himayat, and Y. Li, "Energy-efficient link adaptation in frequency-selective channels," IEEE Trans. Commun., vol. 58, no. 2, pp. 545-554, Feb. 2010.

[5] — "Energy-efficient transmission in frequency-selective channels," in Proc. IEEE Globecom 2008, Nov. 2008, pp. 1-5.

[6] G. W. Miao, N. Himayat, Y. Li, and D. Bormann, "Energy-efficient design in wireless OFDMA," in Proc. IEEE ICC 2008, May 2008, pp. 3307-3312.

[7] F. Richter, A.J. Fehske and G.P. Fettweis, "Energy efficiency aspects of base station deployment strategies for cellular networks," in 2009 IEEE 70th Vehicular Technology Conference Fall (VTC 2009-Fall), Sep. 2009, pp. 1-5.

[8] Y. Chen, S. Zhang, S. Xu and G. Y. Li, "Fundamental trade-offs on green wireless networks," IEEE Commun. Magazine, pp. 30-37, Jun. 2011.

[9] G. W. Miao, N. Himayat, G. Y. Li, and S. Talwar, "Low-complexity energy-efficient OFDMA," in Proc. IEEE ICC 2009, Jun. 2009, pp. $1-5$.

[10] - , "Low-complexity energy-efficient scheduling for uplink OFDMA," IEEE Trans. Commun., vol. 60, no. 1, pp. 112-120, Jan. 2012.

[11] G. W. Miao, N. Himayat, G. Y. Li, A. T. Koc, and S. Talwar, "Interference-aware energy-efficient power optimization," in IEEE 2009 International Conference on Communications, Dresden , Germany, Jun. 2009.

[12] G. W. Miao, N. Himayat, G. Y. Li, and S. Talwar, "Distributed interference-aware energy-efficient power optimization," IEEE Trans. Wireless Commun., vol. 10, no. 4, pp. 1323-1333, Apr. 2011.

[13] Y. Nam, L. Liu, G. W. Miao, C. Zhang, "Link adaptation for energyefficient uplink coordinated multi-point receptions," EURASIP Journal on Wireless Communications, vol. 2011, no. 1, p. 184, 20011.

[14] N. Guo, R. Qiu, S. Mo, and K. Takahashi, "60-GHz millimeter-wave radio: principle, technology, and new results," EURASIP Journal on Wireless Communications and Networking, pp. 1-1, Jan. 2007.

[15] F. M. Mohammed, R. N. Razooqi, and O. F. Abdullah, "Determinations of the relation between the attenuation coefficients of $\mathrm{x}$-ray for copper and some of its alloys with grain size," European Journal of Scientific Research, vol. 45, no. 2, pp. 176-189, 2010.

[16] John M. Senior, Optical Fiber Communications Principles and Practice 3rd. Pearson Education Limited., 2009.

[17] S. K. Yong and C. C. Chong, "An overview of multigigabit wireless through millimeter wave technology: potentials and technical challenges," EURASIP J. Wireless Commun. Networking, 2007, article ID: 78907. 